\title{
A Proposal Approach for Enhanced Route Stability in MANET Using AODV Protocol
}

\author{
Sonia Salaria ${ }^{\mathrm{a}}$, Anil Sagar ${ }^{\mathrm{b}}$, Baljinder Singh $^{\mathrm{c}}$ \\ ${ }^{a}$ Research Scholar, Beant College of Engineering and Technology, Gurdaspur, Punjab, India \\ ${ }^{\mathrm{b}}$ Beant College of Engineering and Technology, Gurdaspur, Punjab, India \\ ${ }^{c}$ Beant College of Engineering and Technology, Gurdaspur, Punjab, India \\ asatbirkaur019@gmail.com
}

\begin{abstract}
A MANET is an autonomous collection of mobile users that communicate over relatively bandwidth restricted wireless links. Since the nodes in topology are mobile, the network might change rapidly and unpredictably with time. Due to mobility of nodes in a network, the path stability is disturbed and hence the link breakage occurs. In this paper, an estimated time based stable routing methodology is proposed with the help of flowchart, which further will improve breakage of link and hence the lifetime of the network will increase considerably.
\end{abstract}

$* * * * *$

\section{Introduction}

In wireless systems, PCs speak with one another through a non-target medium, utilizing radio waves. They can organize a wireless system to work in Basic Service (BSS) mode or in Independent Base Services (IBSS). In BSS mode or in interchanges mode, cell phones speak with one another by means of a non-versatile Base Station (BS) as a passageway. IBSS or MANET modes, don't enable point-topoint correspondence of wireless gadgets, which means they needn't bother with a passageway or any settled framework. A specially appointed system comprises of wireless gadgets that can be portable and equipped for imparting without the requirement for a settled framework. In a MANET, since nodes can move in sporadic ways, the system topology can change vigorously. Nodes can move at any speed toward any path and join or leave the system whenever. Nodes with different limits (e.g., transmission interim) can exist together. A specially appointed portable system (MANET), otherwise called impromptu wireless system is a system of cell phones that self-arranges persistently and without foundation. Every gadget in a MANET is allowed to move freely toward any path and consequently will normally change its associations with different gadgets. Everybody should advance the traffic not identified with their utilization and, subsequently, be a switch. The primary test in the development of a MANET is to give every gadget to always keep up the data fundamental to accurately course the traffic. These systems can work alone or can be associated with the bigger Internet. They can contain at least one diverse handsets bettheyen the nodes. This converts into an incredibly unique and free topology.

The next section discuses about the literature survey, based on which the problem will be formulated and a deterministic solution will be shown with the help of flowchart in the $3^{\text {rd }}$ section of this paper.

\section{Related Work}

[1] Founded the quantity of MANET routing conventions and argued about the functionality of number. Our convention consider centers around Perkins' work to create and improve MANET routing, from old conventions like DSDV to further developed conventions like MAODV. A progression of distributions identified with the MANET directing field has been perceived and changed; they additionally look at the writing on the theme of AETVbased MANET insurance, as this could be the most famous MANET convention. The literature review distinguished various patterns inside research work, for example, the selective utilization of the arbitrary waypoint portability demonstrate, barring key measurements of reenactment results and not contrasting convention execution and accessible options.

[2] Said that the Mobile Adhocsystem is a system in which the nodes convey with no focal organization or system structure. They are interconnected by wireless methods and can utilize different bounces to change information with them. Routing conventions are essential for correspondence and organization in such specially appointed systems, where the productive and auspicious conveyance of the message is coordinated. The DSR is a basic and effective routing convention planned explicitly for use in specially appointed wireless systems with various bounces of portable nodes. DSR permits the system without the requirement for any current foundation or system organization.

[3] Said that specially appointed versatile systems (MANET) are foundation less portable systems that don't have fixed routers. All nodes can move and can be 
batteryfully associated self-assertively utilizing radio waves. This record centers on the investigation of specially appointed versatile systems (MANET'S) and their characterization and qualities. This report additionally centers on the issues and difficulties that are required for specially appointed versatile systems (MANET'S).

[4] Said that a specially appointed portable system (MANET) is made out of versatile nodes (MNs) with no fix foundation. At present, it is a standout amongst the most intriguing research area in wireless communication. These MNs batteryfully make an impermanent system and exchange messages from one $\mathrm{MN}$ to others similarly. A routing convention keeps running on every versatile host and is along these lines subject to asset confinement on every node. Along these lines, so as to ensure correspondence, an effective directing system is attractive, enabling the nodes to impart in a convenient way. This routing method ought to almost certainly limit estimation overhead on the versatile host just as over-burdening traffic on the system. In this record, survey the MANET and late routing conventions for productive correspondence.

[5] Founded that data theory systems are utilized to derive analytical articulations for the base expected length of control messages traded by proactive directing in an impromptu two-level progressive system. A few entropy measures are acquainted and utilized with connection the memory estimate required for putting away the routing tables. The entropy rates of the topology groupings are utilized to connect the correspondence directing overburden, both the inward routing over-burden inside a gathering and the outer directing over-burden over the gatherings. The investigation of the scaling of the quiet over-burden is given in connection to the quantity of nodes and the extent of the bunch in three diverse methods for resizing the system. At long last, down to earth plan issues are examined by giving ideal group sizes that asymptotically lessen (I) the memory necessities for each bunch head; (ii) the over-burden of the absolute directing of the control messages.

[6] Said that specially appointed portable systems (MANETS) can be characterized as systems that have many free and self-made nodes, in any case, these nodes are associated with one another as equivalents. DSR (Dynamic Source Routing) is an on-request directing convention for specially appointed wireless systems that frames a path on interest when a transmission node asks for another. DSRs are utilized to diminish flooding of routing demands. Be that as it may, as the system develops bigger, node versatility and the nearby reserve of each stored way of the portable nodes rapidly turned out to be out of date or wasteful. In this paper, for a productive hunt, researchers have proposed a conventional inquiry calculation in the association of acquainted reserve memory to look all the more rapidly single/different ways for the goal, on the off chance that it exists in the store of the middle of the road versatile node with intricacy $\mathrm{O}(\mathrm{n})$ ( where $\mathrm{n}$ is the quantity of bits) is required to speak to the sought field). The other serious issue with the DSR is that the way support instrument does not locally reestablish a broken connection and out of date reserved data could likewise produce irregularities amid the revelation/remaking way. Thus, to take care of this issue, authors proposed a reserving the executives conspire upgraded for the on-request routing convention (DSR).

[7] Focused on preparing kNN questions in specially appointed portable systems (MANET). Key difficulties in structuring framework conventions for MANET incorporate minimal effort adjustment ability for system topology changes because of node versatility and inquiry handling that accomplishes high question result precision without a server concentrated. In this archive, they propose the fill zone technique (FA) to proficiently process $\mathrm{kNN}$ questions in MANET. In the FA strategy, the information components stay in the nodes close to the situations with which the components are bound, and the nodes reserve information components whose positions are near yours so the sender of the inquiry recovers $\mathrm{kNNs}$ of neighboring nodes. Through broad recreations, authors checked that their proposed methodology accomplishes a low overhead and a high precision of the inquiry result.

[8] Said that the hereditary calculation is one of the incredible assets for setting up research and improvement strategies. Consistent or discrete sections can be improved. Among the met heuristics as of now accessible, the developmental calculation can locate the best capacity with the constrained accessible information accessible. The gathering in Mobile Adhoc systems has picked up significance among scientists to encourage the support of the system. Bunching will be sufficient for compelling correspondence of gathering systems, for example, fiasco help systems, and so forth. The issue in the MANET group is the successive re-connection of the bunch and the absence of system steadiness. In this paper, they propose a way to deal with discover the soundness of the system utilizing a hereditary calculation dependent on the normal number of groups, load adjusting factors and theyighted parameters. The best target work for system soundness is grouped utilizing the hereditary calculation. The test results demonstrate that the soundness of the system can be boosted by deciding the normal number of groupings shaped amid transmission and the heap adjusting component of the bunch head and the recently theyighted parameters decided before transmission.

[9] Said that the genuine reachable execution of MANET, specifically the execution of such systems under viable 
requirements, is still to a great extent obscure. As a stage forward in this sense, this report centers around a MANET where a most extreme conveyance delay is forced on each bundle and looks at the effect of this deferred conveyance limitation on its real execution attainable as far as execution and pack from start to finish. As a matter of first importance, authors decided the execution limit of MANET to uncover the greatest conceivable execution that the system can bolster. For an exogenous speed given to every node, they give study of the execution possible, the bundle conveyance report and the start to finish deferral of the packet, and show how they are dictated by the steady status of the hand-off lines in the system. For the investigation of the steady condition of the retransmission lines, they have additionally built up an unpretentious Markov chain display dependent on state decrease, so the issue of the blast of the state space in the study can be stayed away from. At long last, the numerical outcomes are exhibited to show the execution of the system and the effect created by the constraint of the conveyance delay.

\section{Architecture for Proposed Approach}

Based on the above-related work, an observation is made that Estimated time based stable routing is better and efficient mechanism for improving the link breakage in MANETs. Below figure shows the working of the mechanism:

\section{Flowchart}

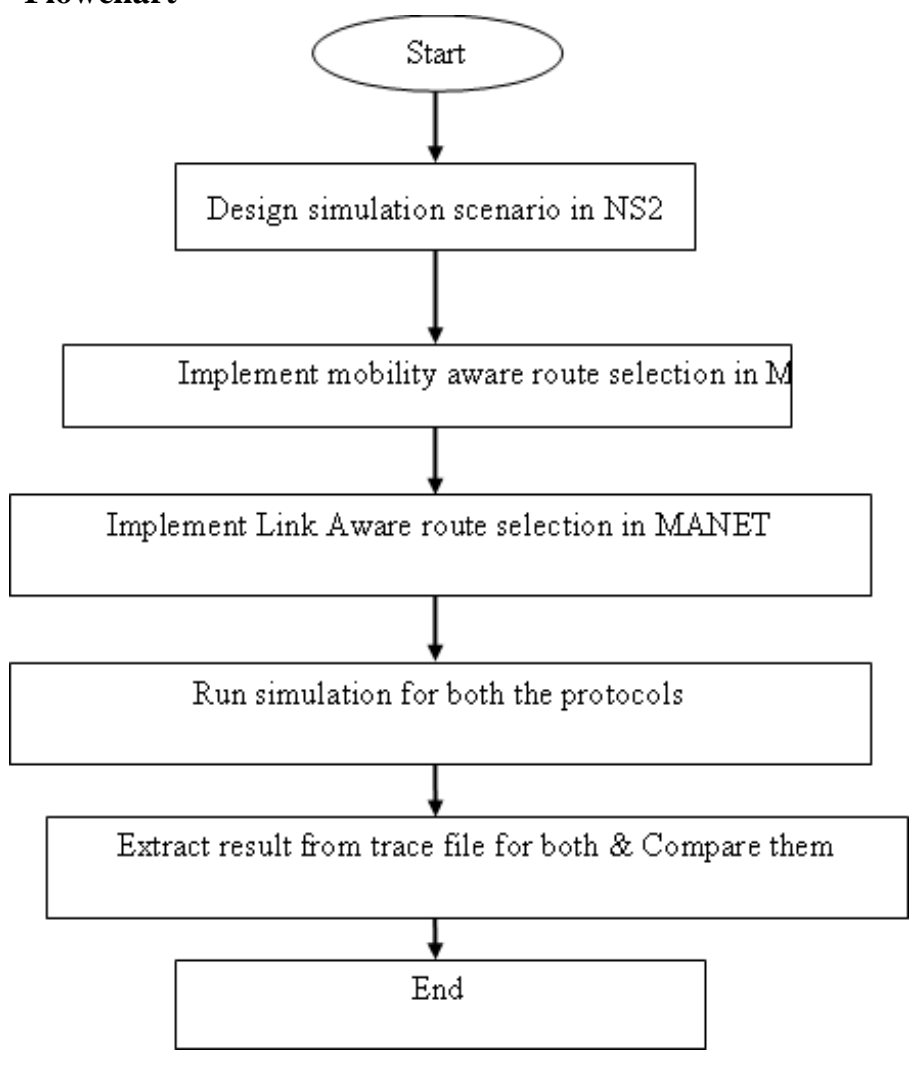

Fig. 1. Proposed Flowchart

\section{Conclusion}

MANETs can be placed, where it is reasonably impossible to set up and defend a wired network. In this research work, the signal strength is continuously measured till the node go out of range. Then, time is noticed and if time limit is below threshold value, then the path is not selected. On the other hand, if the value of time limit is above threshold, the path is selected and hence the less breakage occurs in that case.

\section{References}

[1] Hinds, M. Ngulube, S.Zhu and H. Al-Aqrabi, "A Review of Routing Protocols for Mobile Ad-Hoc NETworks (MANET)," International Journal of Information and Education Technology, Vol. 3, No. 1, 2013.

[2] C.Singh, Vikas Gupta , Gurmeet Kaur, "A Review Paper on Introduction to MANET," International Journal of Engineering Trends and Technology (IJETT) - Vol. 11, No. $1,2014$.

[3] Mohsin U.R Salfi, "A study of Mobile Ad-hoc NetworksIssues and Challenges," International Journal of Advanced Research in Computer Science, Vol. 6, No. 7, 2015.

[4] S. Habib, S. Saleem and K. Muhammad Saqib, "Review on MANET Routing Protocols and Challenges," 2013 IEEE Student Conference on Research and Development (SCOReD), 2013, Putrajaya, Malaysia.

[5] N. Zhou, A. A. Abouzeid, "Routing in Ad Hoc Networks: A Theoretical Framework with Practical Implications," IEEE, 2005.

[6] A. A. Anchari, A. Amin, S. Ashraf, "Routing Problems in Mobile Ad hoc Networks (MANETs)," A Monthly Journal of Computer Science and Information Technology, IJCSMC, Vol. 6, Issue, 2017.

[7] Y. Komai, Y.SasakiI, (Member, IEEE), T. Hara, (Senior Member, IEEE), and S.Nishio, (Fellow, IEEE), "k Nearest Neighbor Search for Location-Dependent Sensor Data in MANETs," 2015.

[8] V. Preetha and K. Chitra, "Prediction of stability of the clusters in Manet using Genetic Algorithm," IEEE International Conference on Advances in Computer applications (ICACA), 2016. 